Supporting Information

\title{
Superhalogen Species of Titanium Oxide Related Clusters Generated by Laser Ablation
}

Shen Bian ${ }^{1}$, Yuan $\mathrm{Ma}^{1}$, Yingying Shi ${ }^{1}$, Xinting Fan ${ }^{1}$, Xianglei Kong ${ }^{1,2 *}$

${ }^{1}$ The State Key Laboratory of Elemento-organic Chemistry, Collage of Chemistry, Nankai University, Tianjin, 300071, China.

${ }^{2}$ Collaborative Innovation Center of Chemical Science and Engineering, Nankai

University, Tianjin, 300071, China

Corresponding to Prof.X. Kong, E-mail: kongxianglei@nankai.edu.cn 
Table S1 The energy difference of $b_{3}^{-}, d_{3}^{-}, f_{3}^{-}, b_{4}^{-}, d_{4}^{-}, d_{5}^{-}$, at different spin multiplicities, obtained at the level of B3LYP/Lanl2dz/ 6-311++G(2d,2p). The corresponding geometries of these isomers are shown in the Fig. 2 in the paper

\begin{tabular}{cccc}
\hline anions & \multicolumn{3}{c}{$\Delta \mathrm{E}$} \\
\hline $\mathrm{b}_{3}^{-}$ & singlet & triplet & quintet \\
\cline { 2 - 4 } $\mathrm{d}_{3-}^{-}$ & 0 & 7.81 & 11.09 \\
$\mathrm{f}_{3}^{-}$ & 0 & 3.41 & 6.90 \\
$\mathrm{~b}_{4}^{-}$ & 0 & 3.11 & 3.11 \\
$\mathrm{~d}_{4}^{-}$ & 0 & Optimization failed & Optimization failed \\
$\mathrm{d}_{5}^{-}$ & 0 & 2.30 & Optimization failed \\
\hline
\end{tabular}

Table S2 The energy difference of $b_{3}, d_{3}, f_{3}, b_{4}, d_{4}, d_{5}$ at different spin multiplicities, obtained at the level of B3LYP/Lanl2dz/6-311++G(2d,2p). The corresponding geometries of these isomers are shown in the Fig. 3 in the paper

\begin{tabular}{cccc}
\hline neutral & \multicolumn{3}{c}{$\Delta \mathrm{E}$} \\
\hline & doublet & quartet & quintet \\
\cline { 2 - 4 } $\mathrm{b}_{3}$ & 0 & Optimization failed & 6.2 \\
$\mathrm{~d}_{3}$ & 0 & 3.22 & 6.62 \\
$\mathrm{f}_{3}$ & 0 & 2.69 & Optimization failed
\end{tabular}




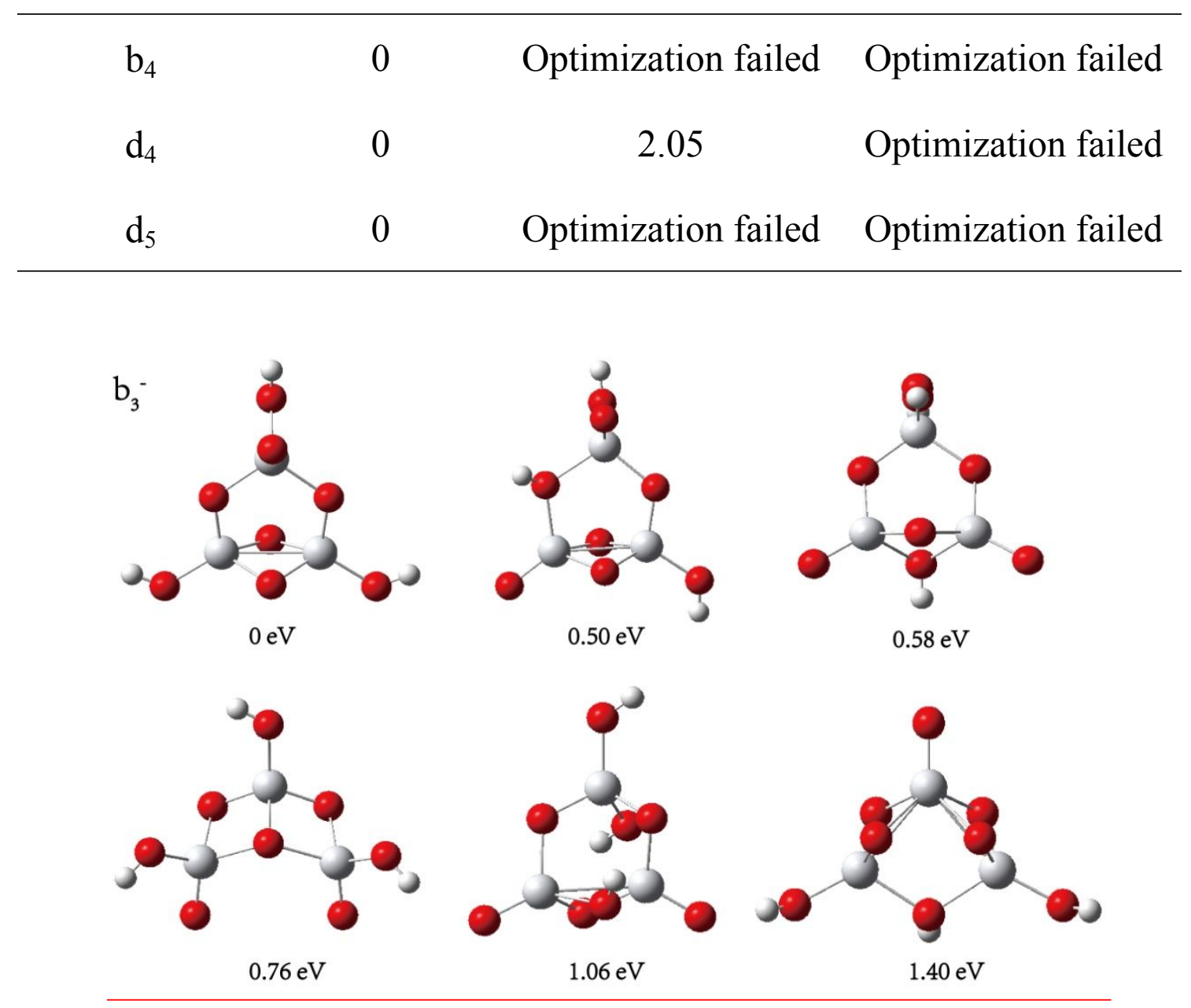

Figure S1. Some optimized isomers of $b_{3}{ }^{-}$, and their relative energies.

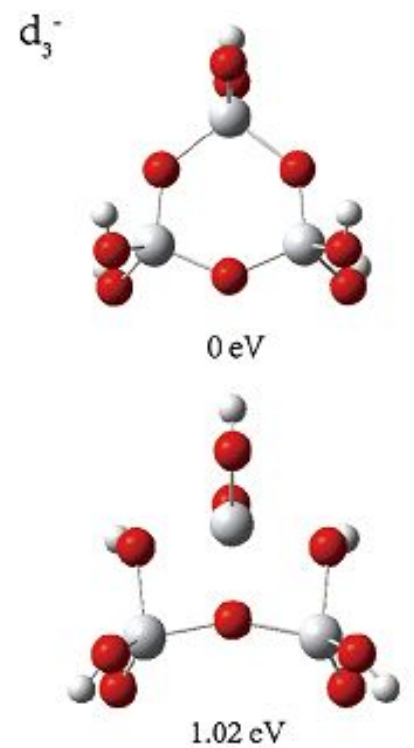

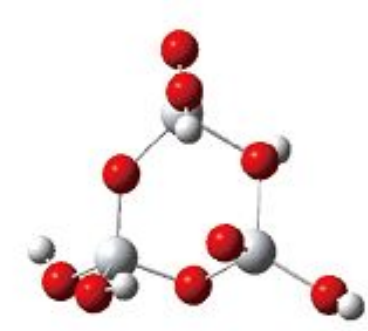

$0.57 \mathrm{eV}$

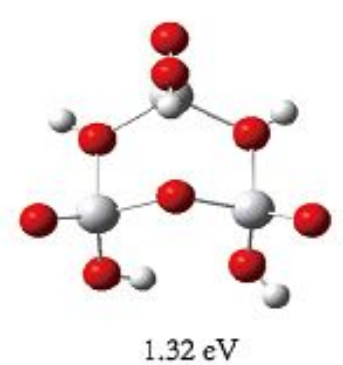

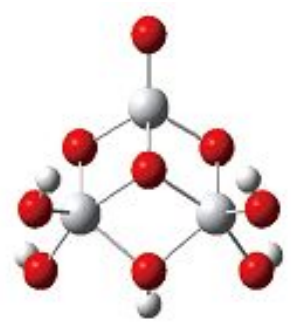

$0.75 \mathrm{eV}$

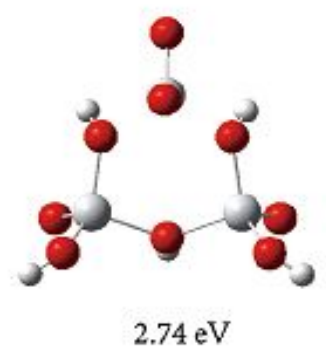

Figure S2. Some optimized isomers of $\mathrm{d}_{3}{ }^{-}$, and their relative energies. 


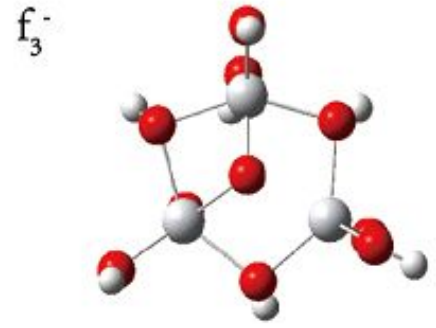

$0 \mathrm{eV}$

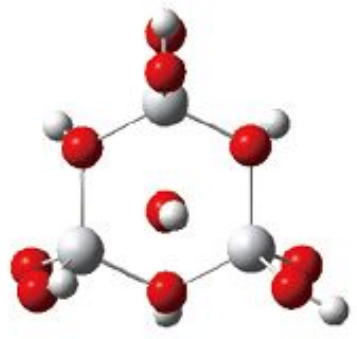

$0.08 \mathrm{eV}$

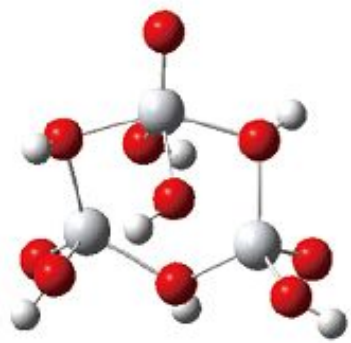

$0.48 \mathrm{eV}$

Figure S3. Some optimized isomers of $f_{3}^{-}$, and their relative energies.

$\mathrm{b}_{4}^{-}$
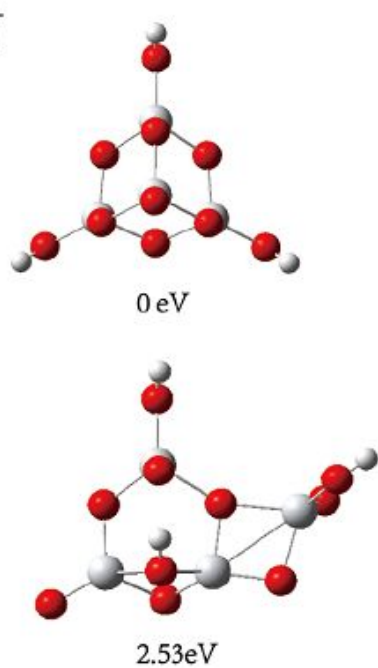
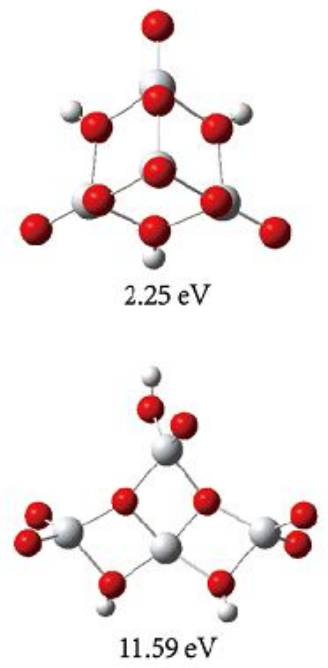

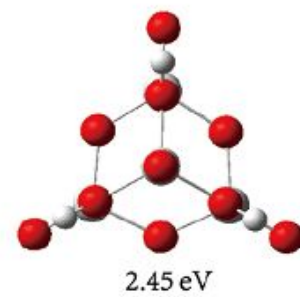

$2.45 \mathrm{eV}$

Figure S4. Some optimized isomers $b_{4}^{-}$, and their relative energies.
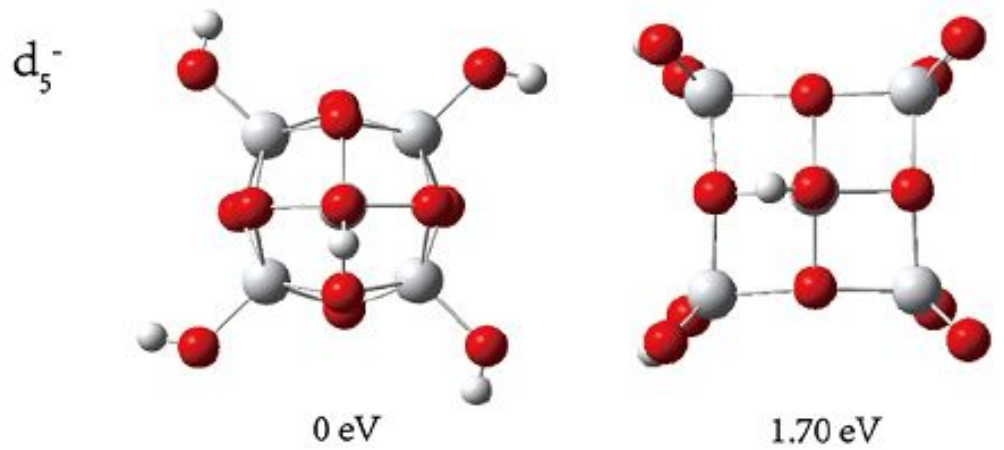

Figure S5. Some optimized isomers of $\mathrm{d}_{5}{ }^{-}$, and their relative energies. 


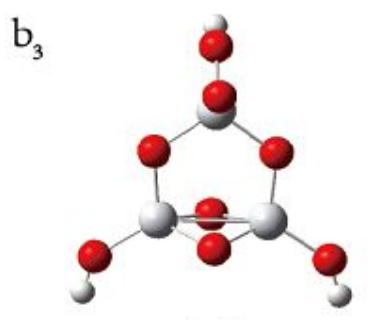

$0 \mathrm{eV}$

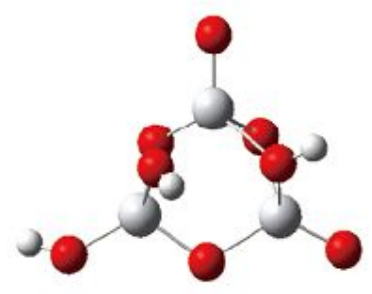

$1.38 \mathrm{eV}$

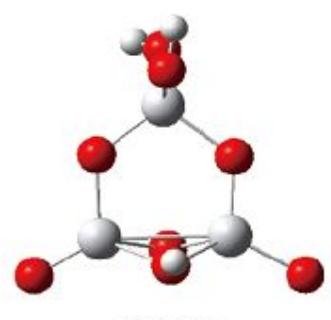

$0.86 \mathrm{eV}$

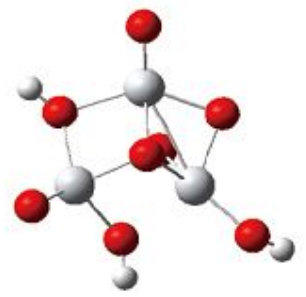

$1.71 \mathrm{eV}$

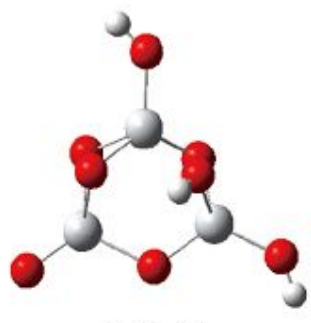

$1.05 \mathrm{eV}$

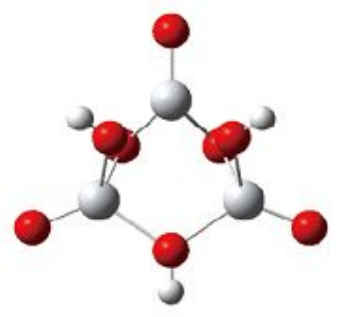

$2.05 \mathrm{eV}$

Figure S6. Some optimized isomers of $b_{3}$, and their relative energies.

$\mathrm{d}_{3}$
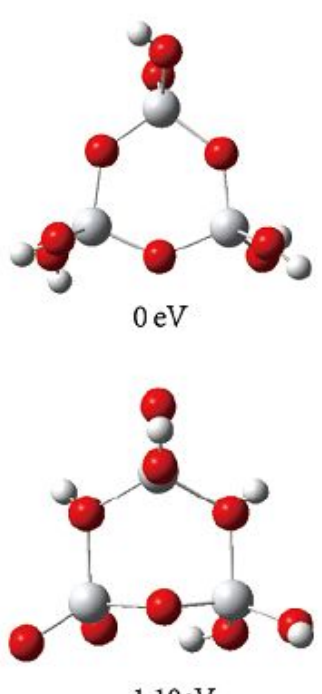

$1.19 \mathrm{eV}$
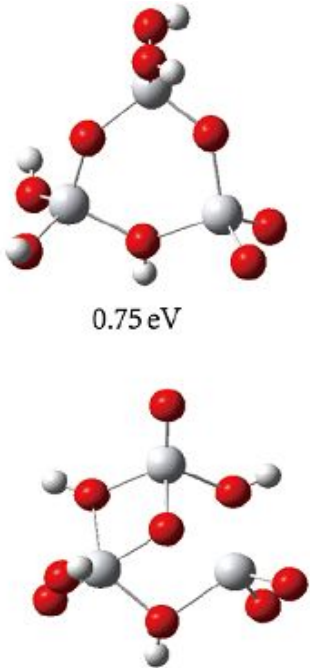

$1.51 \mathrm{eV}$
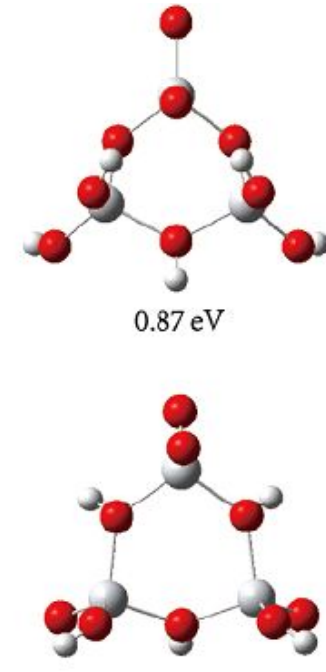

$1.82 \mathrm{eV}$

Figure S7. Some optimized isomers of $\mathrm{d}_{3}$, and their relative energies.

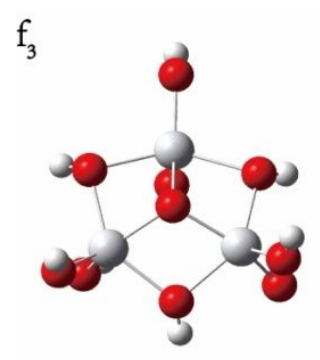

$0 \mathrm{eV}$

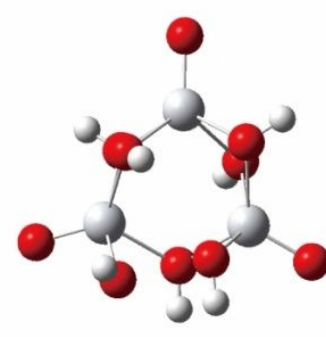

$1.02 \mathrm{eV}$

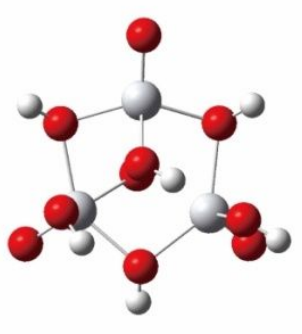

$1.08 \mathrm{eV}$

Figure S8. Some optimized isomers of $\mathrm{f}_{3}$, and their relative energies. 
$\mathrm{b}_{4}$
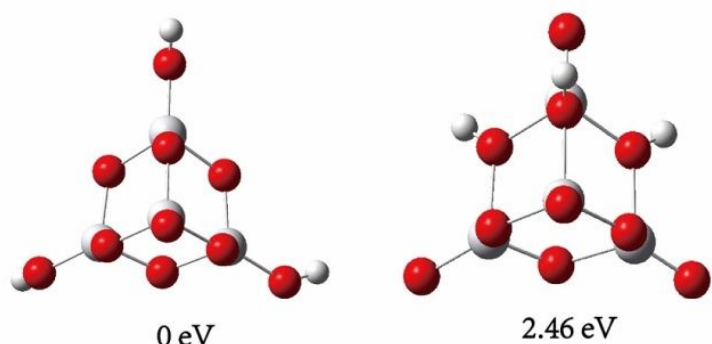

$2.46 \mathrm{eV}$

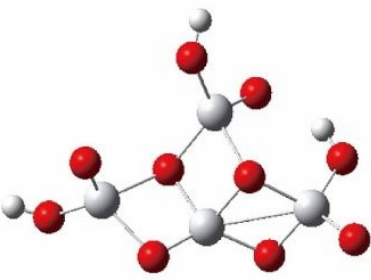

$3.00 \mathrm{eV}$

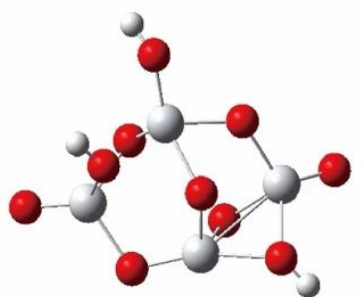

$3.20 \mathrm{eV}$

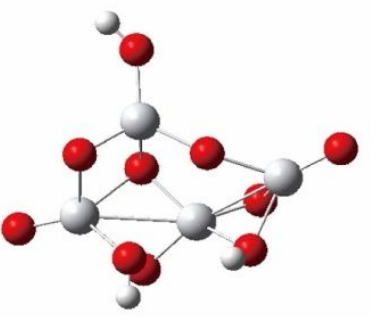

$3.56 \mathrm{eV}$

Figure S9. Some optimized isomers of $b_{4}$, and their relative energies.

$d_{5}$

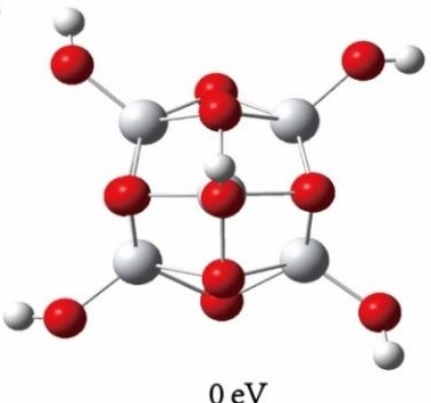

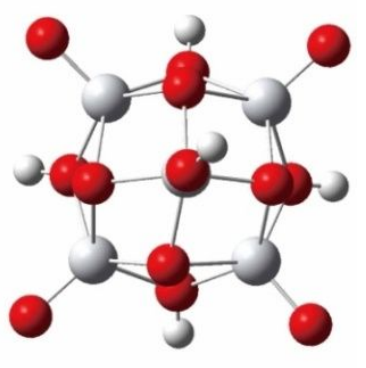

$1.32 \mathrm{eV}$

Figure S10. Some optimized isomers of $\mathrm{d}_{5}$, and their relative energies. 